\title{
Surface parameters from SEVIRI observations through a Kalman filter approach: application and evaluation of the scheme in Southern Italy
}

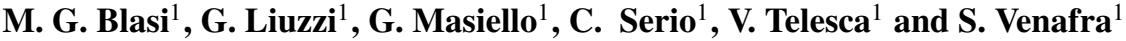 \\ ${ }^{1}$ School of Engineering, University of Basilicata, Italy \\ Received: 12-V-2015 - Accepted: 21-I-2016 - Original version
}

Correspondence to: sara.venafra@unibas.it

\begin{abstract}
Geostationary satellites are capable of resolving the diurnal cycle by providing time sequence of observations with a very high temporal resolution. A Kalman filter methodology was developed to exploit such time continuity in order to simultaneously retrieve surface temperature and emissivity from SEVIRI (Spinning Enhanced Visible and Infrared Imager) data. The methodology was applied and tested over a geographic region in Southern Italy characterized by different surface features: arid, cultivated, vegetated and urban areas, and sea water. The objective is to implement a real-time continuous monitoring of surface parameters, which could be used for the various purposes of tourism and agronomy, land surveillance, natural hazards and risk assessment analysis. The retrieval of surface parameters was performed for the whole of 2013 and the results were compared to other similar satellite observations such as those derived from AVHRR (Advanced Very High Resolution Radiometer) and MODIS (Moderate Resolution Imaging Spectroradiometer). Comparisons with ECMWF (European Centre for Medium range Weather Forecasts) analyses for sea surface are provided as well.
\end{abstract}

Key words: SEVIRI infrared observations, Kalman filter, physical retrieval, surface parameters, real-time monitoring

\section{Introduction}

Information about surface properties, such as temperature and emissivity are important for climate change assessment analysis, risk management and bio-meteorology, to name a few. Infrared instruments on-board geostationary satellites, like SEVIRI on MSG (Meteosat Second Generation), are reaching the accuracy and the spectral quality of sensors on board polar-orbiting platforms (Hilton et al., 2012; Masiello et al., 2011). Compared to polar-satellite instrumentation, geostationary sensors are potentially able to solve the diurnal cycle and provide time series of data whose temporal continuity yields a valuable constraint, which can be exploited for the simultaneous retrieval of surface temperature $\left(T_{s}\right)$ and emissivity $(\epsilon)$. For this purpose, a Kalman filter (KF) methodology (Kalman, 1960; Kalman and Bucy, 1961) was designed and implemented (Amoroso et al., 2013; Masiello et al., 2013) for $T_{s}-\epsilon$ separation and estimation from MSG SEVIRI infrared radiances.
Until now, $T_{s}-\epsilon$ retrieval approaches from geostationary satellites have focused on multispectral data but without considering multitemporal information (Li et al. (2013)). In contrast, our KF approach (Masiello et al., 2013, 2015) is one of the very few physical-based methods for the estimation of surface parameters, which simultaneously retrieves $T_{s}-\epsilon$ and takes advantage of the time continuity provided by the geostationary satellites. A multispectral, multitemporal, physical-based strategy for geostationary observations was also developed in $\mathrm{Li}$ et al. (2011). These schemes are normally much more demanding in terms of computing time and hardware resources than simple statistical methods, which are therefore more attractive in case of real time applications or in situations where the hardware resources are limited.

The main objective of this paper is to show the feasibility of the KF, physical-based methodology for the development of a prototype processor for the real-time retrieval of surface parameters from SEVIRI radiances and 
in case we are dealing with a limited regional area. The target area of interest is that of the Basilicata district in Southern Italy. However, we stress that methodology and results are quite general and, in principle, the KF approach can be applied to any kind of geographical area from the regional to the global scale (Masiello et al. (2013, 2015)). The application to the local scale is very attractive because both the amount of SEVIRI data and computing time are large enough to still permit a real time processing of observations. This characteristic makes the processor very attractive for applications in different environmental fields such as the early warning and detection of forest fires, the over-viewing and monitoring of pollution (oil spills, trash vortex, to name a few), the monitoring of the bio-activity of plants, cultivated areas and the estimation of the water deficit (Sanchez et al. (2008); Copertino et al. (2012); Scavone et al. (2014)). The scheme can be easily incorporated into an operational framework as available in public, regional, environmental and protection agencies.

The present KF methodology has already been validated for sea and land surface, e.g. Masiello et al. (2015). A further assessment of the retrieval performance of the scheme will also be provided in this paper.

In particular, $T_{s}-\epsilon$ sea surface retrievals obtained by $\mathrm{KF}$ application from a suitable target area in Southern Italy will be compared to equivalent observations from AVHRR (Advanced Very High Resolution Radiometer) and MODIS (Moderate Resolution Imaging Spectroradiometer) sensors. Comparison with ECMWF (European Centre for Medium range Weather Forecasts) analysis will be provided, as well. The comparison considers a time slot of one year in order to show that the methodology can correctly follow and retrieve the diurnal and seasonal cycles. However, as already stressed, the present study focuses on the feasibility of KF to run in real-time at the scale of the Mediterranean basin. For a full assessment of the retrieval performance of the scheme, we refer intersted readeres to Masiello et al. (2013, 2015).

This paper is organized as follows. Section 2 deals with the data used in the analysis. In section 3 a summary of the retrieval methodology is presented. Section 4 will show and describe retrieval results. Conclusions are drawn in section 5.

\section{Data}

For the purpose of this paper, SEVIRI Meteosat-10 high rate level 1.5 image data have been acquired for the whole of 2013 for the target area shown in Fig. 1.

This target area extends from $39.25^{\circ}$ to $42^{\circ}$ North latitude and from $13^{\circ}$ to $18.75^{\circ}$ East longitude including both sea and land surface, for a total of 9643 SEVIRI pixels. Ancillary data provided by ECMWF analysis have also been used in order to characterize the thermodynamical atmospheric state. These products contain data about surface temperature $T_{s}$, atmospheric temperature profile $(T)$, specific humidity $(Q)$ and ozone $(O)$. Profiles are available on 91 pressure levels

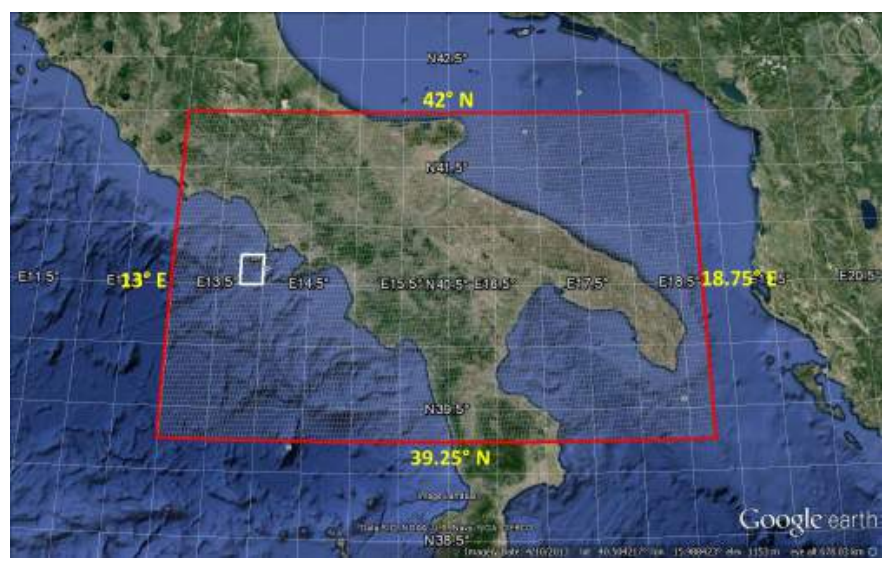

Figure 1. Google map of the target area; the gridded area corresponds to the SEVIRI pixels used for the analysis. The white box shows the area used to compare SEVIRI to AVHRR sea surface products.

until June 252013 and 137 pressure levels from June 26 2013. These data are provided on a $0.125^{\circ} \times 0.125^{\circ}$ grid box at the 00:00, 06:00, 12:00 and 18:00 UTC and they are then interpolated to the SEVIRI time-space grid observations to initialize the Kalman filter.

Another important issue concerns the background information used to constrain emissivity retrievals. For this objective, for land surface, the University of Wisconsin Baseline Fit Global Infrared Land Surface Emissivity Database (UW/BFEMIS database, http://cimss.ssec.wisc. edu/iremis/, Borbas and Ruston (2010); Seemann et al. (2008)) was used. This database is available from 2003 to 2012 with a spatial resolution of $0.05^{\circ}$, derived from the monthly mean operational Aqua/MODIS data (called MYD11C3) by means of a conceptual model called the Baseline Fit method developed from laboratory measurements of surface emissivity. Further details about the UW/BFEMIS emissivity transformation into the SEVIRI channel emissivity are reported in Masiello et al. (2014); Serio et al. (2013, 2014).

For the sea surface, Masuda's model (Masuda, 2006) has been used to properly constrain emissivity. In this case, the emissivity state vector was estimated for a given VZA (Viewing Zenith Angle) ranging from $0^{\circ}$ to $89^{\circ}$ and a wind speed from 0 to $15 \mathrm{~m} \mathrm{~s}^{-1}$ with a step size of $1^{\circ}$ and $1 \mathrm{~m} \mathrm{~s}^{-1}$, respectively. The resulting data set was used to define the background vector and the corresponding covariance matrix.

For the purpose of comparison, MODIS and AVHRR products were acquired. MODIS products are Level 2 data, produced and distributed by the NASA Goddard Space Flight Centers Ocean Data Processing System (ODPS), available at the following web site http://oceandata.sci.gsfc.nasa.gov. Both MODIS platforms AQUA and TERRA were used for the comparison. AVHRR data are instead the result of OI (Optimal Interpolation) SST (Sea Surface Temperature) analysis, which merges AVHRR, buoys and ships data 
to convey daily averages. These products are available at the web link ftp://eclipse.ncdc.noaa.gov/pub/OI-daily-v2/ $\mathrm{NetCDF} /$, distributed over a regular space grid of $0.25^{\circ}$ $\times 0.25^{\circ}$.

\section{Method}

As previously mentioned, emissivity and surface temperature are simultaneously retrieved with a Kalman filter approach. In this methodology, the retrieved state vector consists of $m=8$ SEVIRI infrared channel emissivities and surface temperature,

$\mathbf{v}=\left(e_{1}, e_{2}, \ldots, e_{m}, T_{s}\right)^{T}$

where the superscript $T$ stands for transpose and $e$ is the logit transformed emissivity:

$e=\log \frac{\epsilon}{1-\epsilon}$

In principle, the scheme could be applied to the $m=8$ infrared channels of the SEVIRI imager; their nominal definition is listed in Table 1.

\begin{tabular}{cc}
\hline SEVIRI channel number & nominal wave length $(\mu \mathrm{m})$ \\
\hline 4 & 3.9 \\
5 & 6.2 \\
6 & 7.3 \\
7 & 8.7 \\
8 & 9.7 \\
9 & 10.8 \\
10 & 12.0 \\
11 & 13.4 \\
\hline
\end{tabular}

Table 1. SEVIRI infrared channels

However, since we are interested in surface parameters, the scheme could lead to reliable results only working with the radiances corresponding to atmospheric windows (channels 7,9 and 10) which are marginally affected by the atmospheric parameters, namely temperature, water vapour and ozone.

The usage of a short wave window channel at $3.9 \mu \mathrm{m}$ for retrieval purposes is recommended only at nighttime, to avoid solar contamination and other non-LTE effects.

The non-linear Kalman Filter estimate or analysis, $\hat{\mathbf{v}}_{\mathbf{t}}$ of $\mathbf{v}_{t}$ at a generic time $t$ can be expressed in this way (Nychka and Anderson, 2010):

$\hat{\mathbf{x}}_{t}=\mathbf{x}_{t a}+\left(\mathbf{K}_{t}^{T} \mathbf{S}_{\varepsilon}^{-1} \mathbf{K}_{t}+\mathbf{S}_{a}^{-1}\right)^{-1} \mathbf{K}_{t}^{T} \mathbf{S}_{\varepsilon}^{-1}\left(\mathbf{y}_{t}-\mathbf{K}_{t} \mathbf{x}_{t a}\right)(3)$

where we use the following notation

$\left\{\begin{array}{c}\hat{\mathbf{x}}_{t}=\hat{\mathbf{v}}_{t}-\mathbf{v}_{t ; f g} \\ \mathbf{y}_{t}=\mathbf{R}_{t}-F\left(\mathbf{v}_{t ; f g}\right) \\ \mathbf{x}_{t a}=\mathbf{v}_{t a}-\mathbf{v}_{t ; f g}\end{array}\right.$

The subscripts $a$ and $f g$ indicate background and first guess parameters at time $t$, respectively. $\mathbf{R}$ is the observed radiance vector and $F$ is the function describing the forward radiative transfer model. Again, with reference to Eq. (3), $\mathbf{K}_{t}$ is the Jacobian at time $t$,

$\mathbf{K}_{t}=\left.\frac{\partial F(\mathbf{v})}{\partial \mathbf{v}}\right|_{\mathbf{v}=\mathbf{v}_{t ; f g}}$

The a-posterior covariance matrix for the estimate (3) at time $t$ is given by

$\hat{\mathbf{S}}_{t}=\left(\mathbf{K}_{t}^{T} \mathbf{S}_{\varepsilon}^{-1} \mathbf{K}_{t}+\mathbf{S}_{a}^{-1}\right)^{-1}$

The analysis is propagated forward to time $t+1$ according to the general Kalman Filter theory, yielding the forecast parameters vector and the associated covariance matrix:

$\hat{\mathbf{v}}_{t+1}^{f}=\mathbf{M} \hat{\mathbf{v}}_{t}$

$\hat{\mathbf{S}}_{t}^{f}=\mathbf{M} \hat{\mathbf{S}}_{t} \mathbf{M}^{T}+\mathbf{S}_{\eta}$

The dynamical model operator $\mathbf{M}$ describes the spatiotemporal evolution of the parameter vectors. In this work, it is assumed to be the identity matrix (Masiello et al. (2013), Masiello et al. (2015)). In this way, the retrieval scheme assumes a persistence model for both surface temperature and emissivity. Although it could seem physically inadequate, such a choice makes the scheme a data-driven system. In the previous equations, the covariance matrices $\mathbf{S}_{\varepsilon}, \mathbf{S}_{a}, \mathbf{S}_{\eta}$ are the observational covariance matrix, the background covariance matrix and the stochastic term covariance matrix, respectively. $\mathbf{S}_{\varepsilon}$ is a static matrix, whose diagonal is equal to the SEVIRI radiometric noise. As already stated, for land surface emissivity, $\mathbf{S}_{a}$ is obtained from the UW/BFEMIS database, whereas for sea surface, it is defined and derived according to Masuda's emissivity model. In the end, $\mathbf{S}_{\eta}$ is a tuning stochastic matrix of the filter which trades off between observations and dynamical model.

It can be shown (Nychka and Anderson, 2010) that the analysis derived via Eq. (3) is the minimizer of the linearized quadratic form or cost function $S$ given by

$$
\begin{aligned}
S= & \min _{\mathbf{x}} \frac{1}{2}\left(\mathbf{y}_{t}-\mathbf{K}_{t} \mathbf{x}_{t}\right)^{T} \mathbf{S}_{\varepsilon}^{-1}\left(\mathbf{y}_{t}-\mathbf{K}_{t} \mathbf{x}_{t}\right)+ \\
& \frac{1}{2}\left(\mathbf{x}_{t}-\mathbf{x}_{t a}\right)^{T} \mathbf{S}_{a}^{-1}\left(\mathbf{x}_{t}-\mathbf{x}_{t a}\right)
\end{aligned}
$$

which says that the KF equations (3) and (6) are the same as the equations of Optimal Estimation (Rodgers, 2000). Optimal Estimation can be regarded as a particular case of Kalman Filter (Nychka and Anderson, 2010).

\section{Results}

In this section, the KF retrieval results over the target area (Fig. 1) are presented and described. 

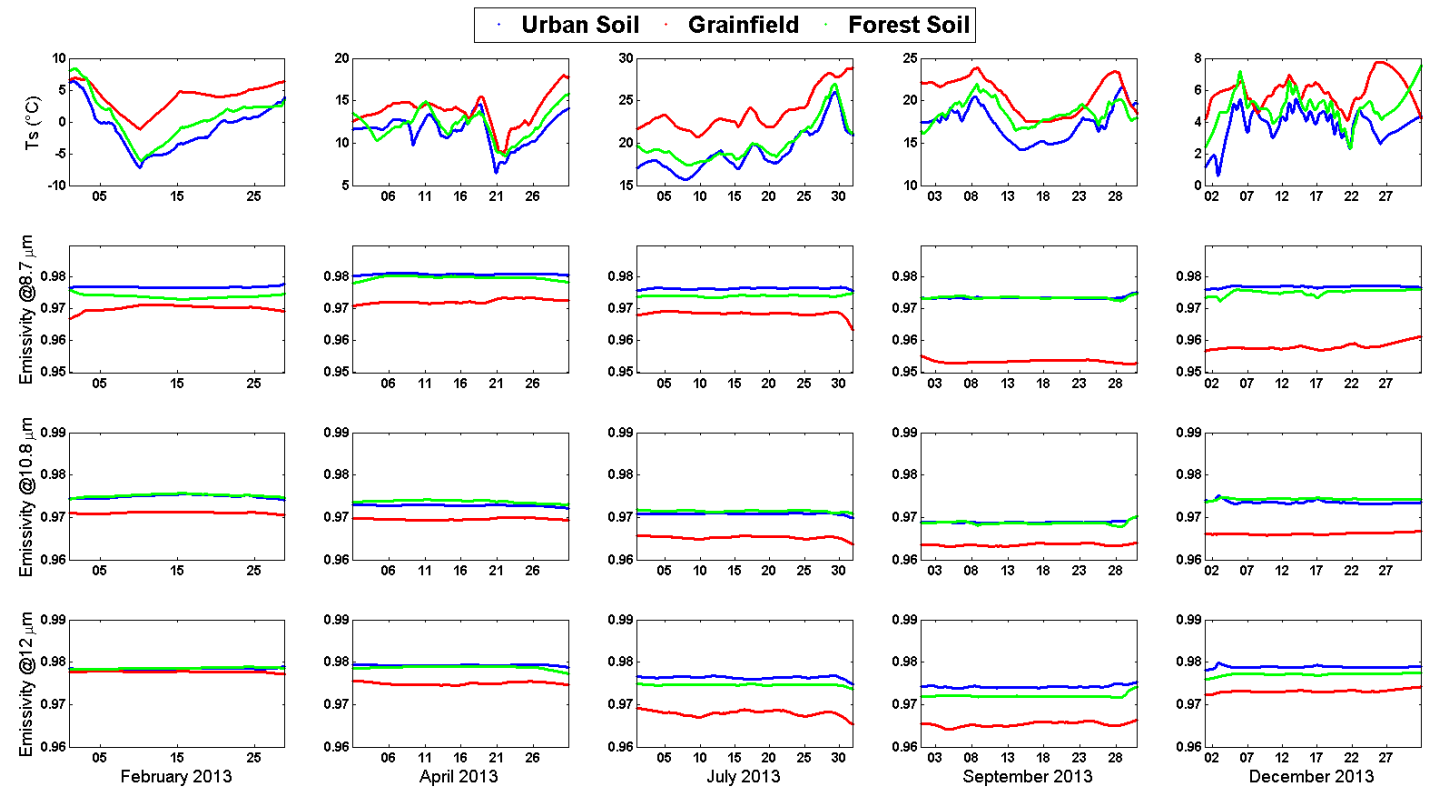

Figure 2. Time sequence of SEVIRI $T_{s}$ and $\epsilon$ for three sites illustrated in Fig. 3.

\section{1 $T_{s}-\epsilon$ retrievals for land surface}

The KF estimation for $T_{s}-\epsilon$ is obtained for clear skies only. For this purpose, we use the operational SEVIRI cloud mask. Cloudy radiances are skipped during the analysis. Because of undetected cloudiness, the KF scheme can occasionally yield unlike values for $T_{s}-\epsilon$, which are filtered out through a suitable, post-processing, smoothing filter. One possible concern with undetected cloudiness and large data voids due to detected clouds, is the stability of KF. However, we have found that our KF implementation yields stable retrievals that are capable of following the seasonal variations of emissivity caused by land cover changes. This capability is exemplified in Fig. 2, which shows the time sequences (15-minute step) of surface temperature and emissivity retrievals for the SEVIRI pixels characterized by surface features as illustrated in Fig. 3.

In the case of grain fields we expect a seasonal variation in emissivity since vegetation is dominated by green grass from January-May (growth), dry grass during June-September (maturity) and finally by bare soil in October-December (decay). This behaviour is correctly shown by emissivity retrieval (see Fig. 2). For urban and forest sites the emissivity is however almost constant with a slight deep during summer due to the dry season.

As mentioned, $\left[T_{s}, \epsilon\right]$ were retrieved for the whole of 2013. For the sake of brevity, only some representative monthly maps are shown here. For land surface, Figs. 4-5 illustrate maps of surface temperature and emissivity averaged over the retrievals corresponding to April and August. It is quite evident that the seasonal cycle is followed very well and the local climate induced by orography and/or land cover is also clearly evidenced in each map.

This is better shown in Fig. 6, where the monthly average retrievals of surface temperature at $00,06,12$ and 18 UTC on August 2013 are illustrated. According to the daily cycle, the retrieved surface temperature reaches higher value during daytime than night-time. The white pixels in Fig. 6 correspond to cloudy scenes.

\section{2 $T_{s}-\epsilon$ retrievals for sea surface}

For sea surface Fig. 7 shows monthly maps of retrieved skin temperature corresponding to January, April, July and October 2013. The spatial distribution of skin temperature displayed in Fig. 7 is in concordance with the known climatology for that area,where the Tyrrhenian Sea is generally warmer than the Adriatic (Cushman-Roisin et al., 2001). Also the colder surface temperature in the Gargano area is in concordance with the formation of dense (colder) water in that area (Cushman-Roisin et al., 2001).

Fig. 8 shows the time sequence of the retrieval for emissivity averaged over the whole target area. As expected, it is seen that the time behaviour shows no trend. The variability is a bit larger in winter than summer, an effect that is likely due to the different cloud coverage between the two seasons and to the subsequent higher frequency of undetected cloudiness in winter with respect to summer.

The retrieved skin temperature was compared to ECMWF, AVHRR and MODIS products. Overall, we found a very good correlation these products both in terms of bias and standard deviation. 

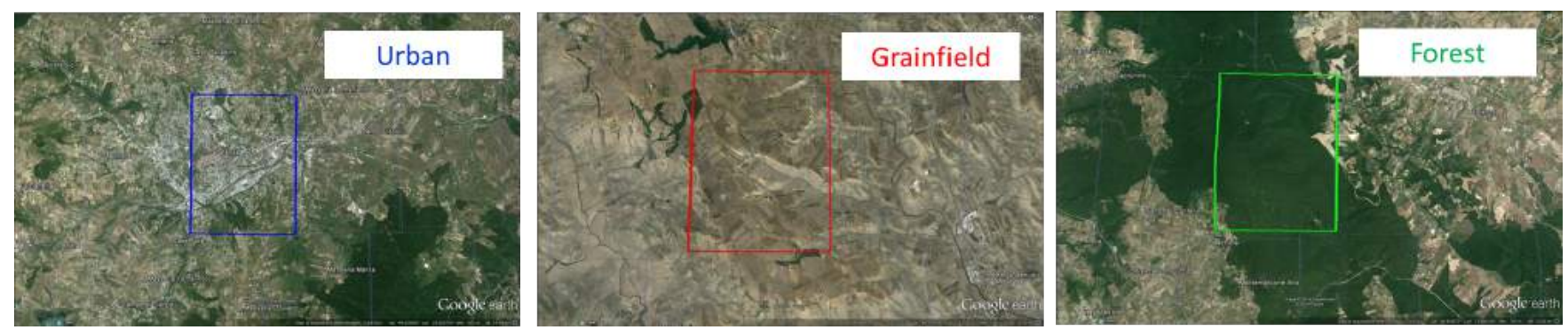

Figure 3. Target areas (urban in blue, grain fields in red and forest in green) whose retrievals are shown in Fig. 2.
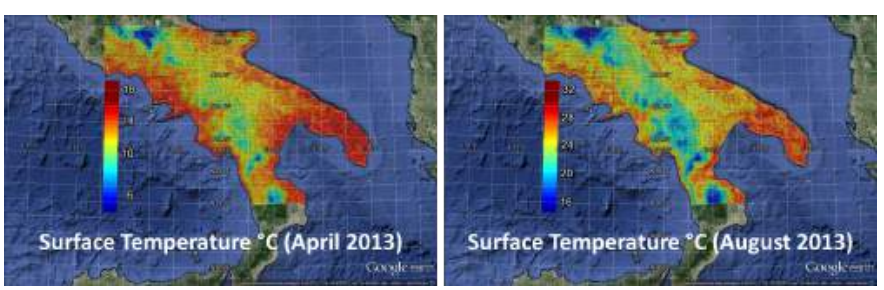

Figure 4. Monthly maps (April on the left-hand-side; August on the right-hand-side) of retrieved land surface temperature for the target area.
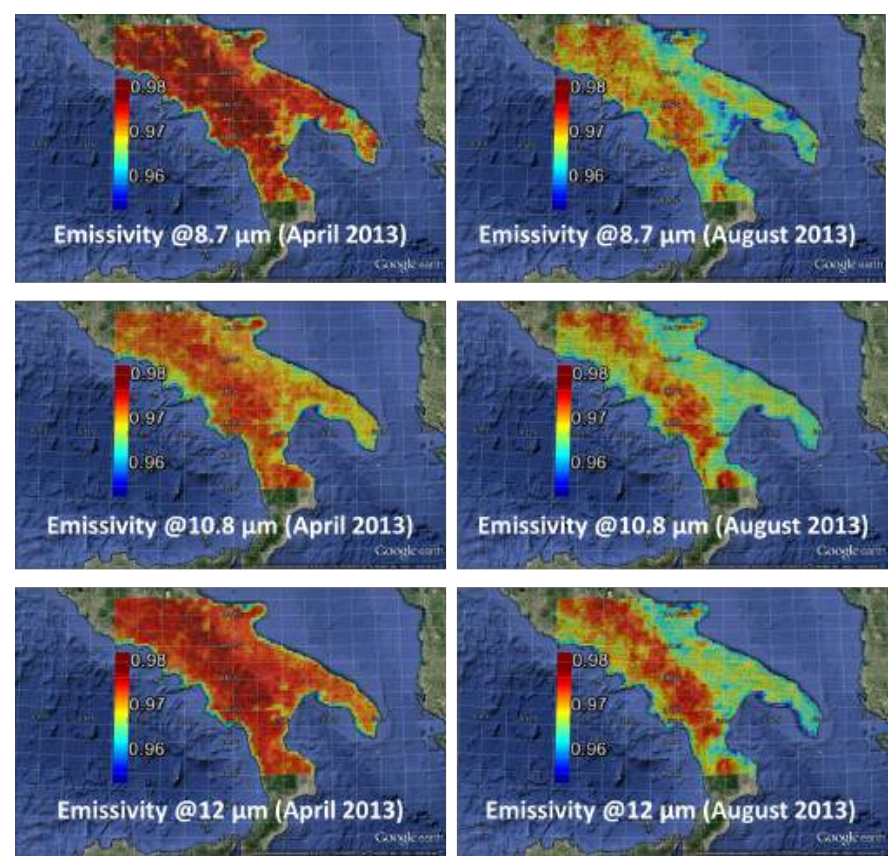

Figure 5. Monthly maps (April, left column; August, right column) for land surface retrieved emissivity for the target area.

The comparison with ECMWF skin temperature yielded absolute monthly mean differences below $0.4{ }^{\circ} \mathrm{C}$ and a standard deviation around $1{ }^{\circ} \mathrm{C}$. When extended to the whole year, the comparison showed a yearly difference of $-0.07{ }^{\circ} \mathrm{C}$ and a standard deviation of about $1.02{ }^{\circ} \mathrm{C}$. The map and the histogram of the skin temperature differences between ECMWF and SEVIRI are shown in Fig. 9. For this last comparison, a number of 2,754,184 pixels have been used.

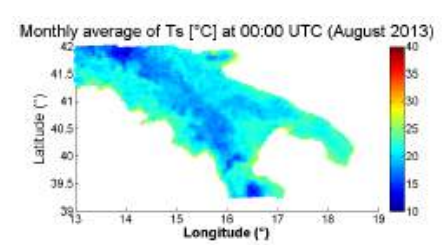

Monthly average of Ts [" $\mathrm{C}]$ at 12:00 UTC (August 2013)
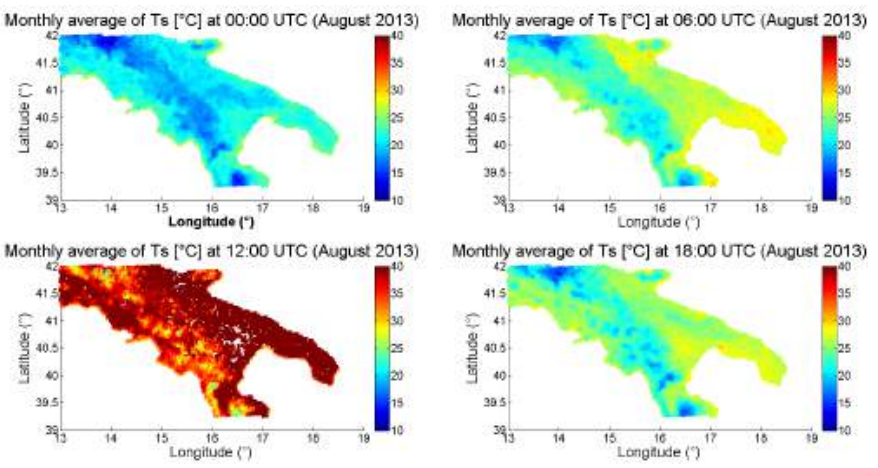

Figure 6. Monthly maps for land surface retrieved temperature at 00, 06, 12 and 18 UTC of August 2013.
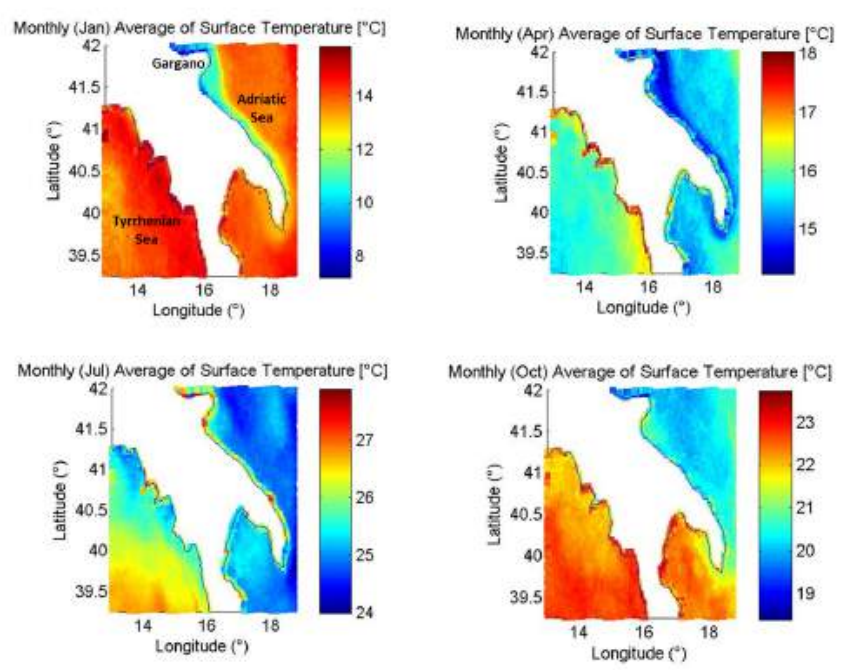

Figure 7. Monthly maps (January, April, July and October 2013) of the retrieved sea skin temperature for the target area.

From Fig. 9(a) it is quite evident that the yearly average difference is homogeneously zero everywhere, except for the SEVIRI pixels near the coasts. With the aim of checking for a seasonal dependence of the difference, the monthly average and the standard deviation were estimated and shown in Fig. 9(c). It is seen that the mean difference and the standard deviation decrease during the summer season. This is quite understandable since the frequency of clouds tends 


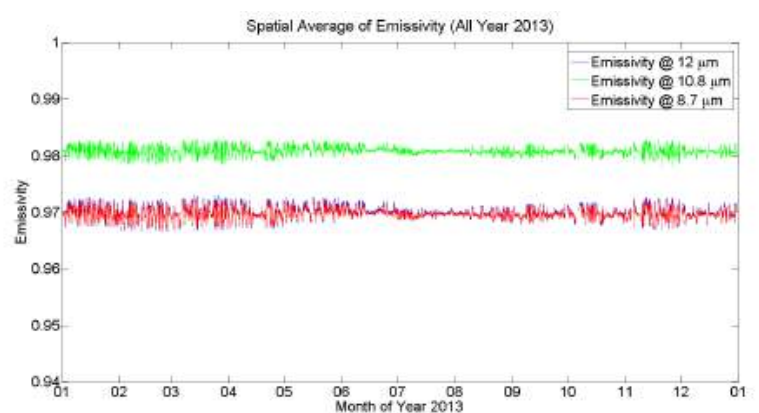

Figure 8. Time sequences of retrieved sea surface emissivity averaged on the whole target area.

to reduce in this season and therefore, more data points can be used for the comparison, which are seemingly less cloud contaminated.

Next, we consider the comparison between SEVIRI and MODIS. As shown in Fig. 10, SEVIRI has a very nice concordance with MODIS; the yearly mean difference is $0.07{ }^{\circ} \mathrm{C}$, whereas the standard deviation is $1.05{ }^{\circ} \mathrm{C}$. These values were obtained based on statistics of 3,230,689 data points.
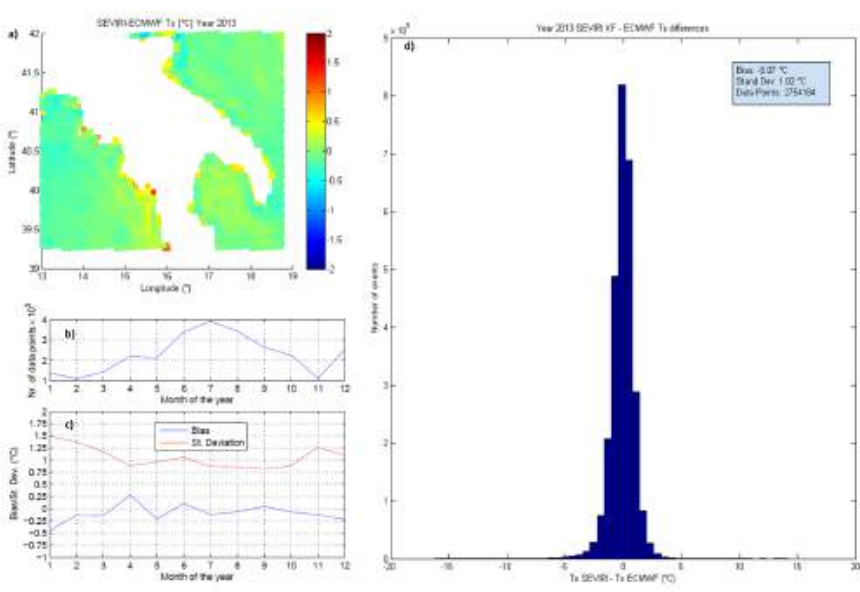

Figure 9. Comparison between SEVIRI KF and ECMWF skin temperature: a) Map of the yearly average difference; b) Number of data point vs month of the year; c) Bias (blue) and standard deviation (red) of the difference; d) Histogram of $T_{s}^{S E V I R I}-T_{s}^{E C M W F}$ differences for the whole year 2013.

The SEVIRI-MODIS comparision was performed using SEVIRI retrieved skin temperatures that were time-space co-located with MODIS overpasses (4 times per day, considering both AQUA and TERRA satellites). Fig. 10(c) shows the monthly mean difference (SEVIRI-MODIS). It is seen that the difference is lower than about $0.2{ }^{\circ} \mathrm{C}$, except for the month of December, for which MODIS shows an offset of about $0.5^{\circ} \mathrm{C}$, a behaviour which is not very well understood at this stage because it is not found for other months.

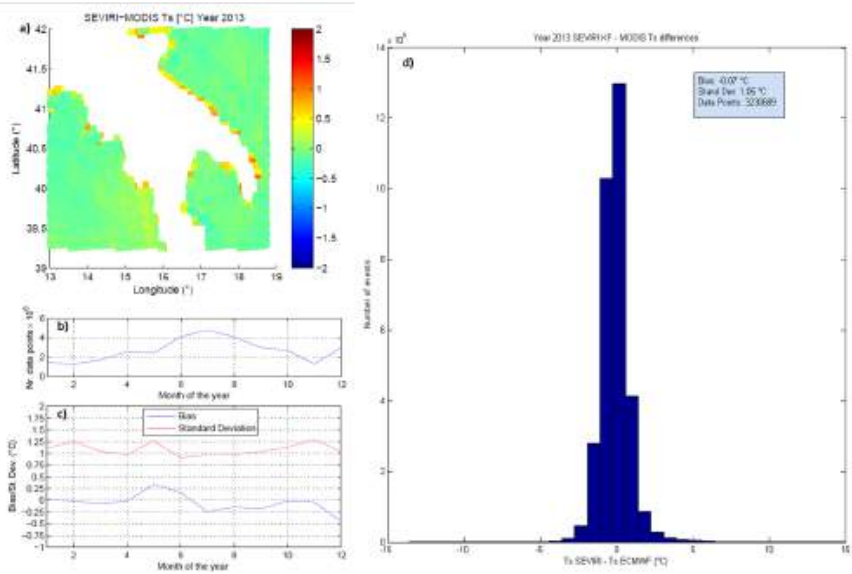

Figure 10. Comparison between SEVIRI KF and MODIS skin temperature: a) Map of the yearly average difference; b) Number of data point vs month of the year; c) Bias (blue) and standard deviation (red) of the difference; d) Histogram of $T_{s}^{S E V I R I}-$ $T_{s}^{M O D I S}$ differences for the whole year 2013 .

Finally, we come to the comparison between AVHRR and SEVIRI sea temperature, which is shown in Fig. 12.

SEVIRI retrieved skin temperature was time-space averaged to be properly compared to AVHRR OI SST data which are available in the form of daily averages on a grid mesh of $0.25^{\circ} \times 0.25^{\circ}$. In the end, the comparison was performed for 3,500 data points. The comparison yielded a bias of $0.27{ }^{\circ} \mathrm{C}$ and a standard deviation of about $0.49^{\circ} \mathrm{C}$. This bias is likely due to the fact that the AVHHR OI SST is a surface temperature whereas our $T_{s}$ is a skin temperature. AVHRR data undergo a de-bias procedure within the OI SST scheme which transforms the satellite skin temperature to that of the surface (Reynolds et al., 2007).

The comparison between SEVIRI and ECMWF, MODIS and AVHRR was also performed for a single pixel in the Tyrrhenian sea (white square box in Fig. 1) located in the Gulf of Naples ( $40.125^{\circ}$ latitude and $13.875^{\circ}$ longitude). The comparison was performed to show that SEVIRI KF $T_{s}$ can follow the yearly cycle. The case SEVIRI-AVHRR is exemplified in Fig. 11 and shows once again a very nice concordance between SEVIRI and AVHRR. There is a systematic bias of about $0.3{ }^{\circ} \mathrm{C}$, which is explained by the aforementioned AVHRR transformation to surface temperature.

\subsection{Performance of the code}

Although based on a fully physical scheme (Amato et al. (2002) and Liuzzi et al. (2015)), which solves and inverts the radiative transfer equation with the accuracy of a monochromatic forward model, the software tool we have developed is very fast. Its computational performance has been tested on a quad core Intel processor with a clock frequency of $2.7 \mathrm{GHz}$ and $1 \mathrm{~GB}$ of RAM. The time needed 

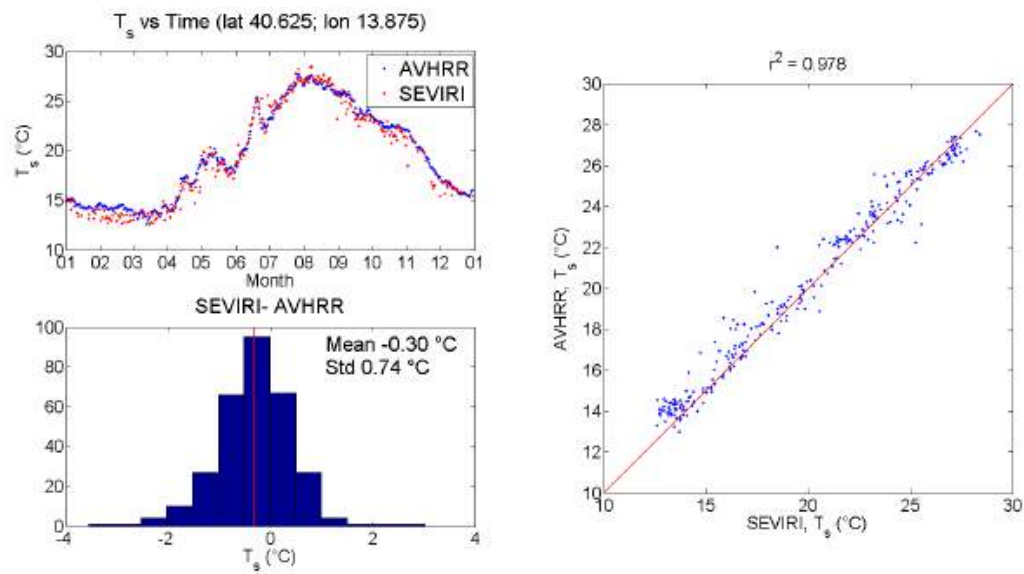

Figure 11. Yearly trend of AVHRR and SEVIRI skin temperatures (top left panel); histogram of differences (bottom left panel) and relative scatter plot of differences (right panel) for one pixel in the Tyrrhenian sea.
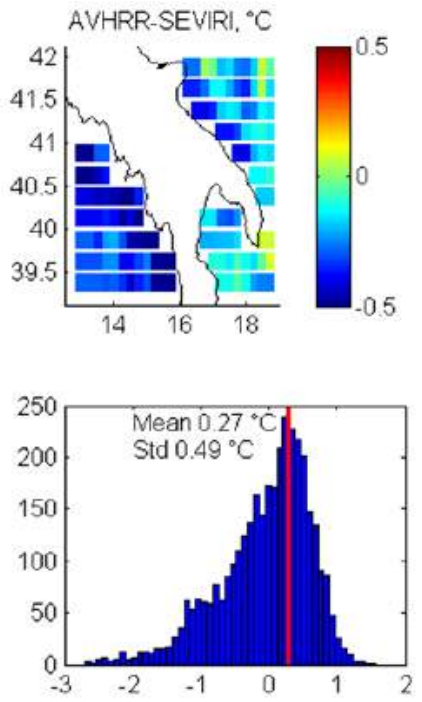

Figure 12. Monthly map (August) AVHRR-SEVIRI skin temperatures difference (top left panel); histogram of differences (bottom left panel) and relative scatter plot of differences (right panel) for target area on sea.

to process one single pixel for a single SEVIRI time slot of $15 \mathrm{~min}$ is $0.04 \mathrm{~s}$. With a single processor the time needed to run over a regional area, e.g., as that shown in Fig. 1 (which is made up of 9643 SEVIRI pixels), would be only 6 min per SEVIRI scene. Since each scene is acquired in a time slot of $15 \mathrm{~min}$, this opens the way to the very first fully physical retrieval scheme for real-time continuous monitoring of surface parameters, which could be used in plenty of practical situations like those already mentioned.

\section{Conclusions}

A Kalman filter methodology was implemented to retrieve skin temperature and emissivity simultaneously from SEVIRI observations. The scheme was demonstrated for a regional target area, encompassing different land cover features, ocean and various weather and climate conditions. Attention was focused on the results obtained over a region of Southern Italy which includes the Basilicata district and the Gulf of Naples. The implementation of the KF approach was evaluated for accuracy and timeliness. It was demonstrated that the code is capable of yielding LST and emissivity retrievals, which follow the local climate induced by orography and/or land cover very well. The retrieval is not affected by the presence of large data voids because of clouds and/or other anomalous events. Good results were also demonstrated for sea surface. For the target area in question, the code can process a single SEVIRI multispectral image (channels at 12.0, 10.8 and $8.7 \mu \mathrm{m}$ ) within six minutes. These values can be scaled to different regions considering that in our case the target area is covered by 9643 SEVIRI pixels. We have shown that a fully physical retrieval scheme can process SEVIRI observations, in realtime and, hence, can be used for continuous monitoring of surface parameters, which could be used for the various purposes of tourism and agronomy, land surveillance, natural hazards and risk assessment analysis. The availability of simultaneous retrievals of surface emissivity and temperature from SEVIRI observations, would improve the exploitation of the European geostationary platforms and also lead to better, improved usage of other European satellite systems.

Acknowledgements. The authors would like to thank EUMETSAT, CNR/MIUR and Regione Basilicata for allowing the development of this work in the framework of the projects EUMETSAT EUM/CO/14/4600001329/PDW, RITMARE (CNR/MIUR), Ricerca ITaliana per il MARE and PO FSE Basilicata 2007-2013 (Atto: D.D. N. 74AB.2013/D.00796 del 02/10/2013 BUR n.38 del 16/10/2013) Decreto n. 300 del 01.07.2014. 


\section{References}

Amato, U., Masiello, G., Serio, C., and Viggiano, M., 2002: The $\sigma$-IASI code for the calculation of infrared atmospheric radiance and its derivatives, Environmental Modelling \& Software, 17, 651-667, doi:10.1016/S1364-8152(02)00027-0.

Amoroso, M., De Feis, I., Masiello, G., Serio, C., Venafra, S., and Watts, P., 2013: Spatio-temporal constraints for emissivity and surface temperature retrieval: Preliminary results and comparisons for SEVIRI and IASI observation, EARSeL eProceedings, 12, 136-148, doi:10.12760/01-2013-2-06.

Borbas, E. E. and Ruston, B. C., 2010: The RTTOV UWiremis IR land surface emissivity module, NWP SAF, EUMETSAT.

Copertino, V., Di Pierro, M., Scavone, G., and Telesca, V., 2012: Comparison of algorithms to retrieve LST from LANDSAT-7 ETM+ IR data in the Basilicata Ionian band, Tethys, 9, 25-34, doi: 10.3369/tethys.2012.9.03.

Cushman-Roisin, B., Gacic, M., Poulain, P. M., and Artegiani, A., 2001: Physical Oceanography of the Adriatic Sea Past, Present and Future, Springer Science+Business Media Dordrecht, doi:10.1007/978-94-015-9819-4.

Hilton, F., Armante, R., August, T., Barnet, C., Bouchard, A., Camy-Peyret, C., Capelle, V., Clarisse, L., Clerbaux, C., Coheur, P. F., Collard, A., Crevoisier, C., Dufour, G., Edwards, D., Faijan, F., Fourrié, N., Gambacorta, A., Goldberg, M., Guidard, V., Hurtmans, D., Illingworth, S., Jacquinet-Husson, N., Kerzenmacher, T., Klaes, D., Lavanant, L., Masiello, G., Matricardi, M., McNally, A., Newman, S., Pavelin, E., Payan, S., Péquignot, E., Peyridieu, S., Phulpin, T., Remedios, J., Schlüssel, P., Serio, C., Strow, L., Stubenrauch, C., Taylor, J., Tobin, D., Wolf, W., and Zhou, D., 2012: Hyperspectral Earth Observation from IASI: four years of accomplishments, B. Am. Meteor. Soc., 93, 347-370, doi:10.1175/BAMS-D-11-00027.1.

Kalman, R. E., 1960: A new approach to linear filtering and prediction problems, Journal of Basic Engineering, 82, 35-45, doi:10.1115/1.3662552.

Kalman, R. E. and Bucy, R. S., 1961: New Results in Linear Filtering and Prediction Theory, Transactions of the ASME - Journal of Basic Engineering, 83, 95-108, doi:10.1115/1.3658902.

Li, J., Li, Z., Jin, X., Schmit, T. J., Zhou, L., and Goldberg, M., 2011: Land surface emissivity from high temporal resolution geostationary infrared imager radiances: 1. Methodology and simulation studies, J. Geophys. Res., 116, doi:10.1029/2010JD014637.

Li, Z.-L., Tang, B.-H., Wu, H., Ren, H., Yan, G., Wan, Z., Isabel, F., Trigo, I. F., and Sobrino, J. A., 2013: Satellite-derived land surface temperature: Current status and perspectives, Remote Sens. Environ., 131, 14-37, doi:10.1016/j.rse.2012.12.008.

Liuzzi, G., Masiello, G., Serio, C., Blasi, M. G., and Venafra, S., 2015: Hyper fast radiative transfer for the physical retrieval of surface parameters from SEVIRI observations, Journal of Physics: Conference Series, 633, doi:10.1088/17426596/633/1/012058.

Masiello, G., Serio, C., and Antonelli, P., 2011: Inversion for atmospheric thermodynamical parameters of IASI data in the principal components space, Quarterly Journal of the Royal Meteorological Society, 138, 103-117, doi:10.1002/qj.909.

Masiello, G., Serio, C., De Feis, I., Amoroso, M., Venafra, S., Trigo, I. F., and Watts, P., 2013: Kalman filter physical retrieval of surface emissivity and temperature from geostationary infrared radiances, Atmos. Meas. Tech., 6, 3613-3634, doi:10.5194/amt6-3613-2013.

Masiello, G., Serio, C., Venafra, S., De Feis, I., and Borbas, E. E., 2014: Diurnal variation in Sahara desert sand emissivity during the dry season from IASI observations, J. Geophys. Res., 119, 1626-1638, doi:10.1002/jgrd.50863.

Masiello, G., Serio, C., Venafra, S., Liuzzi, G., Gottsche, F., Trigo, I. F., and Watts, P., 2015: Kalman filter physical retrieval of surface emissivity and temperature from SEVIRI infrared channels: a validation and inter-comparison study, Atmos. Meas. Tech., 8, 2981-2997, doi:10.5194/amt-8-2981-2015.

Masuda, K., 2006: Infrared sea surface emissivity including multiple reflection effect for isotropic Gaussian slope distribution model, Rem. Sens. Environ., 103, 488-496, doi:10.1016/j.rse.2006.04.011.

Nychka, D. W. and Anderson, J. L., 2010: Data Assimilation. Handbook of Spatial Statistics, Gelfand A., Diggle P., Guttorp P. and Fuentes M.

Reynolds, R. W., Smith, T. M., Liu, C., Chelton, D. B., Casey, K. S., and Schlax, M. G., 2007: Daily High-Resolution-Blended Analyses for Sea Surface Temperature, J. Climate, 20, 54735496, doi:10.1175/2007JCLI1824.1.

Rodgers, C. D., 2000: Inverse methods for atmospheric sounding, Theory and Practice, 2, World Scientific, Singapore.

Sanchez, J., Scavone, G., Caselles, V., Valor, E., Copertino, V., and Telesca, V., 2008: Monitoring daily evapotranspiration at a regional scale from Landsat-TM and ETM+ data: Application to the Basilicata region, J. Hydrol., 351, 58-70, doi:10.1016/j.jhydrol.2007.11.04.

Scavone, G., Sanchez, J., Telesca, V., Caselles, V., Copertino, V., Pastore, V., and Valor, E., 2014: Pixel-oriented land use classification in energy balance modelling, Hydrological Processes, 28, 25-36, doi:10.1002/hyp.9507.

Seemann, S. W., Borbas, E. E., Knuteson, R. O., Stephenson, G. R., and Huang, H. L., 2008: Development of a Global Infrared Land Surface Emissivity Database for Application to Clear Sky Sounding Retrievals from Multispectral Satellite Radiance Measurements, Journal of Applied Meteorology and Climatology, 47, 108-123, doi:10.1175/2007JAMC1590.1.

Serio, C., Masiello, G., Amoroso, M., Venafra, S., Amato, U., and De Feis, I., 2013: Study on Space-Time Constrained Parameter Estimation from Geostationary Data, Final Progress Report, EUMETSAT contract No. EUM/CO/11/4600000996/PDW, EUMETSAT, Darmstadt, Germany, doi:10.13140/RG.2.1.4898.5120.

Serio, C., Liuzzi, G., Masiello, G., Venafra, S., and De Feis, I., 2014: Kalman Filter estimation of surface temperature and emissivity from SEVIRI, Final Report, EUMETSAT contract No. EUM/CO/14/4600001329/PDW, EUMETSAT, Darmstadt, Germany, doi:10.13140/RG.2.1.2017.7442. 